ORIGINAL ARTICLE

\title{
Role of medical students in preventing patient harm and enhancing patient safety
}

\author{
S C Seiden, C Galvan, R Lamm
}

Qual Saf Health Care 2006;15:272-276. doi: 10.1136/qshc.2006.018044

See end of article for authors' affiliations

Correspondence to S C Seiden, University of Chicago, Department of Pediatrics, Comer

Children's Hospital, 5721

South Maryland Avenue, MC8016, Chicago,

IL 60637, USA; seiden@

uchicago.edu

Accepted for publication 6 May 2006
Background: Substantial efforts are focused on the high prevalence of patient harm due to medical errors and the mechanisms to prevent them. The potential role of the medical student as a valuable member of the team in preventing patient harm has, however, often been overlooked.

Methods: Four cases are presented from two US academic health centers in which medical students prevented or were in a position to prevent patient harm from occurring. The authors directly participated in each case.

Results: The types of harm prevented included averting non-sterile conditions, missing medications, mitigating exposure to highly contagious patients, and respecting patients" "do not resuscitate" requests. Conclusion: Medical students are offen overlooked as valuable participants in ensuring patient safety. These cases show that medical students may be an untapped resource for medical error prevention. Medical students should be trained to recognize errors and to speak up when errors occur. Those supervising students should welcome and encourage students to actively communicate observed errors and near misses and should work to eliminate all intimidation by medical hierarchy that can prevent students from being safety advocates.
$\mathrm{D}$ eaths attributed to medical error are estimated to be the third leading cause of death in the US, ${ }^{1}$ resulting in 48 000-98 000 deaths each year. ${ }^{2}$ The literature on preventing medical errors in general is rapidly evolving, but applicability toward trainees has not been sufficiently addressed. Some attention has been paid to the role of resident physicians in preventing errors ${ }^{3-6}$ and to the importance of teaching about patient safety in graduate medical education..$^{7-9}$ However, there has been less attention to the perception of errors by medical students and to the role they could have in error prevention. ${ }^{10-18}$

Medical students want to contribute to the healthcare team, but their lack of clinical knowledge and inexperience (and corresponding lack of confidence), the fact that they are not licensed providers, unwillingness or hesitancy to speak up (possibly routed in fear of negative evaluation or other consequences), and the medical hierarchy all discourage error identification and student communication of observed errors. As the cases in this paper illustrate, student contributions to patient safety therefore may be not initiated, ignored, or overlooked. However, because medical errors are often caused from miscommunication and poor accessibility of information, ${ }^{19-21}$ medical students may be as adept at preventing certain types of errors as other members of the healthcare team. Because students usually follow fewer patients than house staff, they can afford greater attention to details that may frequently lead to medical errors (for example, prescribed versus administered medications). Moreover, the inclusion of medical students in the care of the patient affords increased redundancy to catching medical errors and mitigating their consequences. ${ }^{7}$

Medical students do witness medical errors, with one study reporting that $76 \%$ of medical students had observed a medical error. ${ }^{17}$ Yet only about half of these students (who had received formal training in patient safety) reported the errors to a resident or attending, and only $7 \%$ reported using an electronic error reporting system. These data, and the cases presented below, suggest that substantial cultural changes are needed to make students proactive when they see errors.
This paper illustrates the powerful role medical students can play in preventing patient harm and introduces students as an untapped resource for error prevention. We present four real case histories in which one of the authors (then medical students) prevented or could have prevented patient harm (table 1).

\section{CASES}

Case 1: Sterile technique in the operating room (OR) Case history

A third year medical student on her surgery rotation was scrubbing on a wrist arthroscopy case. The student had seen two previous wrist arthroscopies performed by other surgeons. The resident and student positioned the patient's arm for sterile preparation. After the arm was in position the attending, resident, and student left the OR to scrub. As the patient's arm was being positioned into the traction apparatus the student noticed that skin on the arm of the Caucasian patient lacked the residual yellow hue of the iodine based prep and concluded that the arm must not have been prepped sterilely. The student assumed that the attending was aware of this. On previous surgeries the student had noticed that the surgeons would often double glove while prepping and setting up and then remove their outer gloves. At one point in draping, the patient's unprepped hand touched the attending's arm above his outer gloves. The student mentioned this to the attending but the attending did not respond.

The student felt that the maxim in surgery was "medical students should be seen and not heard" and therefore did not repeat the warning. The student also still thought sterile prep was forthcoming. After the patient's arm was positioned, the student inquired if they should take off their outer gloves (which had been holding the patient's unprepped arm). The resident and attending, confused by the question, responded that the student could. As the attending prepared to make the first incision on the unclean skin, the student quietly told the resident that the patient's arm had not been prepped. The resident replied that the arm had been prepped, but then noticed the patient's arm was still white. The patient's arm 
Table 1 Case examples of student involvement in prevention of medical error

\begin{tabular}{|c|c|c|c|c|}
\hline No & Description of event & Contributing factors or problems & Role of medical student & Lessons learned \\
\hline 1 & $\begin{array}{l}\text { Non-sterile prepped } \\
\text { limb noticed before } \\
\text { incision }\end{array}$ & $\begin{array}{l}\text { Poor team communication } \\
\text { Assumptions of OR staff } \\
\text { responsibilities } \\
\text { OR culture and hierarchy limiting } \\
\text { communication } \\
\text { Daily variation of OR team members }\end{array}$ & $\begin{array}{l}\text { Observations of procedures and deviations } \\
\text { from the norm prompted the student to } \\
\text { question the omission of sterile technique }\end{array}$ & $\begin{array}{l}\text { Don't assume anything; keep a suspect } \\
\text { eye when things do not look correct } \\
\text { Err on the side of caution, err in favor of } \\
\text { the patient } \\
\text { Don't be afraid to speak up, even if you } \\
\text { are wrong } \\
\text { Learn proper sterile techniques }\end{array}$ \\
\hline 2 & $\begin{array}{l}\text { Drugs ordered but } \\
\text { not administered }\end{array}$ & $\begin{array}{l}\text { Drug order system requires } \\
\text { transcription from hand written } \\
\text { order to computer based MAR } \\
\text { No formal practice of confirming } \\
\text { that ordered drugs are administered }\end{array}$ & Drug administration was not confirmed & $\begin{array}{l}\text { Students are in a position to follow the } \\
\text { practice of checking orders are carried } \\
\text { out and medications administered }\end{array}$ \\
\hline 3 & $\begin{array}{l}\text { DNR order not } \\
\text { followed }\end{array}$ & $\begin{array}{l}\text { No system for alerting DNR orders } \\
\text { to team } \\
\text { Student hesitant to communicate } \\
\text { knowledge of DNR order }\end{array}$ & Communicated DNR order to team & $\begin{array}{l}\text { Students encouraged to communicate } \\
\text { with team members when there are } \\
\text { questions about proper procedures }\end{array}$ \\
\hline 4 & Infection exposure & $\begin{array}{l}\text { Language barrier } \\
\text { Emergency setting } \\
\text { Complete history not taken }\end{array}$ & $\begin{array}{l}\text { Student initiated translator consult } \\
\text { Obtained history suggestive of tuberculosis } \\
\text { Initiated respiratory precautions }\end{array}$ & $\begin{array}{l}\text { Effective communication is vital in patient } \\
\text { safety } \\
\text { When language barriers arise, } \\
\text { translators must be used to ensure safe } \\
\text { patient care }\end{array}$ \\
\hline
\end{tabular}

was re-draped, prepped, a sterile traction apparatus located, and the operation proceeded without incident.

\section{Error analysis}

This near miss raises many important issues. As in many medical errors, the etiology was one of poor communication and follow through. The OR team failed to communicate who was going to be responsible for prepping. The student's perception of surgical hierarchy and the OR culture made it difficult for the student to speak up when she first noticed the unprepped arm. The student also felt more comfortable disclosing the error to the resident instead of the attending (a behavior seen elsewhere ${ }^{17}$ ).

In this case the student prevented the medical error. Some students might have spoken up sooner; some may not have said anything at all. In addition, some luck was involved in making this case a near miss (the hue of the iodine based prep on a light skinned patient).

\section{Lessons learned}

The Accreditation Council for Graduate Medical Education (ACGME) competencies for US resident education provide a useful framework for categorizing the lessons learned in the cases in this paper and illustrate how this construct can be applied to undergraduate medical education. ${ }^{22}$ Firstly, the competencies of patient centred care and professionalism suggest that students should prioritize the safety of the patient ahead of their own hesitancy to speak up. However, the student also needs sufficient medical knowledge to recognize that sterile preparation is always performed for invasive procedures. Increased use of interdisciplinary team training (with emphasis on talking to superiors) would enhance the practice-based learning and improvement opportunities, and aim to provide an increased comfort level for any team member to speak up when an error is recognized..$^{23}$

The cornerstone of earlier detection of the near miss in this case rests upon interpersonal and communication skills. Students, as well as the entire healthcare team, should be encouraged to speak up in times of uncertainty regarding procedures and patient safety. Unfortunately, the case above is not an isolated example of a failure to speak up. ${ }^{18}{ }^{24}$ The pre-procedure "time out", which is now mandated by the Joint Commission on Accreditation of Healthcare
Organizations (JCAHO) immediately prior to beginning invasive procedures, ${ }^{25}$ creates a valuable opportunity for communication between members of the operative team. If the team leader uses this "time out" not only for verification of the patient's name and anticipated procedure but also as a forum to remind all operative team members that they are expected to speak up if they see any safety concerns, even junior or novice team members such as medical students may be more likely to express concerns when they arise. Finally, systems based practice changes (such as standardization of the sterile prepping procedure) could provide another approach to prevent this kind of near miss from recurring.

\section{Case 2: Drugs prescribed versus drugs administered Case history}

A student on her first third year clinical rotation was assigned to follow a 21 year old man with steroid treated ulcerative colitis admitted for a planned total abdominal colectomy. The patient was doing well for the first three postoperative days, but on day 4 the student found while pre-rounding that the patient had been vomiting since 3 am and appeared acutely ill. The team later questioned if the symptoms were secondary to the patient's recent change in diet from "nothing by mouth" to clear liquids. A plain abdominal radiograph was ordered and showed apparent free air under the diaphragm, indicating a possible postoperative perforation.

Upon review of the patient's chart the resident realized that the patient had not received the ordered postoperative famotidine (Pepcid) during the first four postoperative days. The service's practice was for selective histamine blockers such as famotidine to be prescribed after gastrointestinal surgery to protect the gastrointestinal mucosa, relaxed from anesthesia, from the potentially erosive effects of gastric acid. While famotidine was written in the postoperative orders, it had never been transcribed to the computer based medication administration record (MAR).

A subsequent gastrointestinal follow through study showed that the patient had not perforated but probably had a postoperative ileus with the observed free air possibly residual from the surgery. He received a nasogastric tube and was finally started on famotidine. The patient quickly improved and was discharged home approximately 4 days later. 


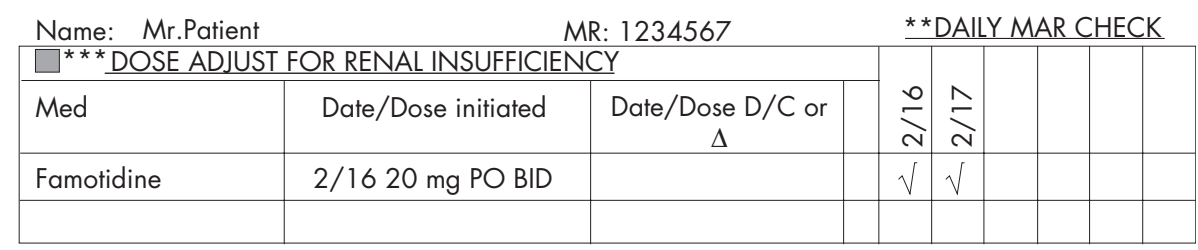

Figure 1 Example of work round checklist to reduce medication errors.

\section{Error analysis}

This case is a relatively benign example of the common problem of medication errors-that is, the failure to administer ordered medications. This medical error could have been prevented in many ways. For example, enhanced systems based practice such as the use of computerized physician order entry systems with standard order sets and decision support may help prevent such errors (although they are not without flaws themselves ${ }^{26}$ ).

\section{Lesson learned}

In this case only the resident noticed the missing medication once the patient became symptomatic. However, this error could have been prevented by the medical student if students performed a daily check of their patients' MAR to see that all medications ordered for their patients are being administered. One suggestion is for students to add a table (fig 1) to their daily work round sheet that includes check boxes for drugs ordered, or to otherwise make it their practice to check the MAR daily. From a competencies perspective, it is also helpful for students' medical knowledge to include common medication errors and drug interactions. Students could also easily be trained to perform the increasingly used safety practice of medication reconciliations, ${ }^{24} 2728$ or to use error prone drugs as "trigger tools" to prompt heightened awareness for error. ${ }^{29}$ Communication skills and professionalism would come into play if the student detected an error.

\section{Case 3: Respecting the do not resuscitate (DNR) order} A third year student on his medicine rotation was following a very sick elderly patient with end stage Alzheimer's disease and severely compromised activities of daily living who was admitted for percutaneous endoscopic gastrotomy tube insertion. The patient's code status was "do not resuscitate" (DNR). On the day of the procedure a cardiac arrest code was called in the post-anesthesia care unit (PACU). Upon arrival in the PACU the resuscitation had been started by the PACU staff, and the student's senior resident began to lead the code. The third year student whispered to a classmate that the patient was DNR but did not know what to do-the student did not know resuscitation efforts could be stopped once initiated. After several minutes the student realized that there was no harm in reminding the team of the DNR status of the patient. After discussion, during which the code continued, resuscitation efforts were terminated and the patient was pronounced dead.

\section{Error analysis}

In spite of the ethical, legal, and individual value of DNR orders, the medical community lacks consistent mechanisms for maintaining continuity of a patient's code status and they have been overlooked in the past. ${ }^{30}$ Wachter and Shojania ${ }^{15}$ reported the termination of resuscitation efforts because the wrong patient's chart was pulled and, similarly, a junior staff member was afraid to speak up (the patient's nurse, just out of nursing school, correctly thought that the patient was full code). This case illustrates the confusion and diffusion of authority that can take place in resuscitations. ${ }^{30}$

\section{Lesson learned}

Like case 1 , this case illustrates the troubling pattern of medical students being afraid to speak up, suggesting greater need for increased communication based competency training of all parties from student to faculty member. When medical students possess vital information it is essential that it is promptly communicated to the team. In addition, students' medical knowledge competency should include information on appropriate initiation and termination of resuscitation efforts. Finally, in terms of patient centred care, emphasis should be placed on appropriate display and communication of patients' individual resuscitation preferences.

\section{Case 4: Infection precautions complicated by language barrier}

A third year student on his medicine rotation was called to the Emergency Department (ED) to evaluate a Spanish speaking patient with a presumed pneumonia. As the student had only moderate Spanish skills, he decided to request a translator. The student found the patient's symptoms included hemoptysis, night sweats, and weight loss, and the patient had recently immigrated from Mexico where her mother had died of tuberculosis (TB). The student immediately obtained protective masks for the providers, the patient, and the patient's husband. The student asked the ED attending whether TB was on her differential diagnosis for this patient, to which the attending responded: "Yeah, I thought about it". The attending did not comment about the possible danger the patient posed to the other patients, the student, or the translator. Examination of the chest radiograph revealed markings consistent with TB. Several days later the patient's sputum tested positive for acid-fast bacilli (diagnostic for TB). The patient was placed on appropriate treatment and exposed staff were screened for PPD conversion.

\section{Error analysis}

The error in this case is one of delaying treatment and exposing other patients and staff to a potentially infectious disease. This error probably occurred because of insufficient triage evaluation of a patient with a cough and was complicated by the language barrier.

\section{Lesson learned}

Medical students are repeatedly told in their training that the patient's history is their most powerful diagnostic tool. An inability to communicate effectively, due to language barrier or otherwise, significantly diminishes the effectiveness of patient histories. Students, with their additional time, may more readily seek the help of translators. In this case we can see that this student's perseverance led to the correct diagnosis and correct treatment for the patient and also avoided further unnecessary exposure of hospital personnel. However, this case also illustrates the difficulty that students and other trainees may encounter when trying to convey information or management suggestions to an unreceptive superior, and the need for competency in communication and patient centred care. In this case the student pursued a diagnosis of TB that the attending had dismissed. In such 
situations, appropriate but sensitive confrontation with the goal of providing safe patient care is imperative. In addition, students should note that systems changes could also improve patient centred care and communication-for instance, by automatically paging a translator when a nonEnglish speaking patient arrives in triage.

\section{DISCUSSION AND RECOMMENDATIONS}

In this paper we present several cases in which medical students appropriately characterized a problematic situation and, in some cases, prevented a medical error and patient harm. Medical students are members of the healthcare team with sufficient knowledge and awareness to recognize medical errors and add another layer to system defences. Moreover, because students follow fewer patients and can spend more time with each patient than residents, they can afford greater attention to detail. While students can and should participate to their fullest ability to enhance patient safety, it should be noted that students should never be seen as being the principal team member responsible for patient safety, as they may often be required to leave patient care activities for lectures, examinations, or to study. And there is always the possibility that a student's knowledge base will be deficient to prevent a particularly complex error from occurring.

This paper does not present examples in which students themselves may cause errors (procedural or otherwise), another important subject that has received only limited attention. ${ }^{15} 31$ Some recommendations-such as a patient safety curriculum for undergraduate medical education, ${ }^{16}{ }^{1732-34}$ the use of interdisciplinary team training, ${ }^{32-40}$ and the use of simulation ${ }^{34} 41^{42}$ - show promise as useful interventions to improve safety, but have been significantly discussed elsewhere. We offer below new recommendations categorized by the ACGME resident based competencies ${ }^{22}$ to increase student awareness of medical errors and to empower them as team members who can contribute to patient safety. These recommendations are derived from the experiences seen in the cases presented above. However, the small number of cases examined may mean that the cases are not generalizable to all medical student experiences.

- Improve students' interpersonal, communication, and professionalism skills. ${ }^{32} 33$ 35-40

- Train students to practise patient centred care and to be familiar with patient information that is essential to safety.

- Train students in elements of practice-based learning and improvement as well as systems-based practice.

- Provide students with appropriate medical knowledge for common causes of medical error.

\section{Recommendation 1: Improve students' interpersonal,} communication, and professionalism skills

Providing health care always involves accessing pertinent information and data. The number of sources and the large volume of information that must be synthesized stack the odds that a clinician may at some point miss an important piece of data. Students should be trained and have practical experience in speaking up without hesitation when they possess information that is critical to safe patient care. The wrist arthroscopy and the DNR order cases indicate the need for students to be vocal when they possess overlooked information and how hierarchy and unfamiliarity with roles and responsibilities can hamper disclosure. Prefacing phrases such as "I'm probably mistaken, but..." or "this might be a ridiculous question, however..." can deflect the confrontational interaction that might otherwise discourage a student from questioning the action of his/her superior.
Similarly, the medical staff hierarchy must become receptive to students raising patient safety-related questions. Students rarely spend a period longer than a month in any one clinical setting, and thus may always have a tendency to feel like an outsider. ${ }^{18}$ Our experience and that of others shows that students may feel hesitant and delay communicating a known error because of their junior or outsider status and the intimidation they feel from the medical hierarchy. ${ }^{34}{ }^{40}$ In aviation, a hesitancy to question the captain led to 583 fatalities in the 1977 collision at Tenerife, Canary Islands-the worst aviation accident in history. As a result of this accident, however, the training technique of crew resource management (CRM) was pioneered in an attempt to teach that concerns regarding safety can and must be conveyed by any team member. ${ }^{15}$ Moreover, high reliability organizations (such as aviation and nuclear power) teach us the importance of having respect for an individual's expertise, regardless of their rank..$^{34}$ In addition, because patient safety is such a new topic in the realm of medical knowledge, all learners from medical student to attending faculty can be considered novices and should be equally receptive to questions and comments regarding safety. ${ }^{43}$

Making the medical staff more receptive to students raising safety questions requires challenging cultural changes that will not occur without consistent high level leadership, ongoing training in communication and teamwork, and accountability for patient safety outcomes. ${ }^{44}{ }^{45}$ In this way the medical staff might see students as assets in trying to help the team reach patient safety goals. Finally, students should recognize that appropriate competency in professionalism means the patient's needs for safe patient care supersede any self-interest that might cause hesitancy in bringing attention to a potential error.

Recommendation 2: Train students to practise patient centred care and to be familiar with patient information that is essential to safety

Students should be familiar with common and essential data about their patients such as medications (that they are ordered and administered), allergies, code status, and other information that might easily be overlooked. The second case above illustrates how an ordered drug may not actually be administered. Also, when patients have a procedure or surgery planned, students should assist the team in completing verification of site, side, correct patient, and correct procedure.

\section{Recommendation 3: Train students in elements of practice-based learning and improvement as well as systems-based practice}

Students should report errors and near miss events to available reporting systems to enhance understanding of errors. ${ }^{174647}$ While students sometimes report errors to residents and faculty, event reporting tools are used much less frequently. ${ }^{17}$ Moreover, by reporting both errors and near miss events, students contribute to the knowledge base that can help prevent future errors and increase their own awareness of error prevention.

In addition, a demonstrated understanding and attention to quality and patient safety should be part of medical student competencies and corresponding evaluation. For medical students especially, assessment drives behavior. ${ }^{34}$ If students are expected to achieve competency in improving quality and safety and are given methods to achieve this competency, they will do so. For example, routine evaluations on clinical clerkships could include a question as to whether the student noticed, discussed, or otherwise contributed to areas of quality improvement or safety concerns. ${ }^{22}$ 


\section{Recommendation 4: Provide students with appropriate medical knowledge for common causes of medical error}

In the case series presented here, medical knowledge was necessary in sterile technique (case 1); postoperative perforations after gastrointestinal surgery and medication prophylaxis (case 2); ethical termination of resuscitation efforts (case 3); and recognition of contagious diseases such as TB (case 4). This is merely an anecdotal list of ways in which medical knowledge can be important to safety. While the necessary knowledge for these cases might be imparted in any medical school curriculum (for example, in the surgery clerkship or ethics course), specific emphasis on how this is safety related content should also be provided. Comprehensive literature on the epidemiology and etiology of medical error should be matched to related content in medical school curricula so that specific safety related medical knowledge is developed. ${ }^{48}$

Incorporation of these recommendations can bolster the safety in academic medical centers by using medical students as a previously untapped advocate for patient safety.

\section{ACKNOWLEDGEMENTS}

The authors thank Julie Johnson, Paul Barach, Robert Wachter, John Hickner, Steve Small, and David Stevens for review and suggestions regarding this manuscript.

\section{Authors' affiliations}

S C Seiden, The University of Chicago, Department of Pediatrics, Comer Children's Hospital, Chicago, IL, USA

C Galvan, Northwestern University, Department of Emergency Medicine, Chicago, IL, USA

R Lamm, Harvard Affiliated Emergency Residency, Brigham and Women's and Massachusetts General Hospital, Boston, MA, USA

Competing interests: none.

\section{REFERENCES}

1 Starfield B. Is US health really the best in the world? JAMA 2000;284:483-5.

2 Kohn LT, Corrigan JM, Donaldson MS, eds. To err is human: building a safer health system. Washington, DC: Institute of Medicine, National Academy Press, 2000.

3 Volpp KG, Grande D. Residents' suggestions for reducing errors in teaching hospitals. N Engl J Med 2003;348:851-5.

4 Hobgood CD, Ma OJ, Swart GL. Emergency medicine resident errors: identification and educational utilization. Acad Emerg Med 2000;7:1317-20.

5 Schenkel SM, Khare RK, Rosenthal MM, et al. Resident perceptions of medical errors in the emergency department. Acad Emerg Med 2003;10:1318-24.

6 Wu AW, Folkman S, McPhee SJ, et al. Do house officers learn from their mistakes? Qual Saf Health Care 2003;12:221-8.

7 Joint Committee of the Group on Resident Affairs and Organization of Resident Representatives. Patient safety and graduate medical education, Association of American Medical Colleges, 2003.

8 Hoff TJ, Pohl H, Bartfield J. Creating a learning environment to produce competent residents: the roles of culture and context. Acad Med 2004;79:532-9.

9 Battles JB, Shea CE. A system of analyzing medical errors to improve GME curricula and programs. Acad Med 2001;76:125-33.

10 Rosebraugh CJ, Tsong Y, Zhou F, et al. Improving the quality of adverse drug reaction reporting by 4 th-year medical students. Pharmacoepidemiol Drug Saf 2003; 12:97-101.

11 Lee SK, Cowie SE. MSJAMA: Medical students and remediation of error. JAMA 2001;286:1082-3.

12 Rajendran PR. MSJAMA: Ethical issues involved in disclosing medical errors. JAMA 2001;286:1078.

13 Fost N. MSJAMA: Ethical issues in whistleblowing. JAMA 2001;286:1079.

14 Wusthoff CJ. MSJAMA: Medical mistakes and disclosure: the role of the medical student. JAMA 2001;286:1080-1.

15 Wachter R, Shojania K. Internal bleeding: the truth behind Americàs terrifying epidemic of medical mistakes, 1st ed. New York, NY: Rugged Land, 2004.

16 Halbach JL, Sullivan LL. Teaching medical students about medical errors and patient safety: evaluation of a required curriculum. Acad Med 2005;80:600-6.

17 Madigosky WS, Headrick LA, Nelson K, et al. Changing and sustaining medical students' knowledge, skills, and attitudes about patient safety and medical fallibility. Acad Med 2006;81:94-101.
18 AHRQ Web M\&M. Low on the totem pole, 2005. Available at http:// webmm.ahrq.gov/case.aspx?caselD = 110 (accessed 1 December 2005).

19 Joint Commission on Accreditation of Healthcare Organizations (JCAHO) Sentinel event statistics, 2005. Available at http://www.jcaho.org/ SentinelEvents/Statistics/ (accessed 10 March 2006)

20 Lingard L, Reznick R, Espin S, et al. Team communications in the operating room: talk patterns, sites of tension, and implications for novices. Acad Med 2002:77:232-7.

21 Sutcliffe KM, Lewton E, Rosenthal MM. Communication failures: an insidious contributor to medical mishaps. Acad Med 2004;79:186-94.

22 Accreditation Council for Graduate Medical Education (ACGME). ACGME outcomes project: general competencies. Version 1.3, 1999. Available at http://www.acgme.org/outcome/ (accessed 1 December 2005).

23 Thomas EJ, Sexton JB, Helmreich RL. Discrepant attitudes about teamwork among critical care nurses and physicians. Crit Care Med 2003;31:956-9.

24 Pronovost $\mathbf{P}$, Weast $B$, Schwarz $M$, et al. Medication reconciliation: a practical tool to reduce the risk of medication errors. J Crit Care 2003;18:201-5.

25 Joint Commission on Accreditation of Healthcare Organizations (JCAHO). Universal protocol for wrong site, wrong procedure and wrong person surgery, 2003. Available at http://www.jointcommission.org/PatientSafety/ UniversalProtocol/ (accessed 15 April 2006).

26 Koppel R, Metlay JP, Cohen A, et al. Role of computerized physician order entry systems in facilitating medication errors. JAMA 2005;293:1197-203.

27 Barnsteiner JH. Medication reconciliation: transfer of medication information across settings - keeping it free from error. Am J Nurs 2005;105(3 Suppl):31-6.

28 Joint Commission on Accreditation of Healthcare Organizations (JCAHO) Joint Commission 2006 National Patient Safety Goals. Available at http:// www.jcaho.org/accredited+organizations/patient+safety/06_npsg_ie.pdf (accessed 15 August 2005).

29 Rozich JD, Haraden CR, Resar RK. Adverse drug event trigger tool: a practical methodology for measuring medication related harm. Qual Saf Health Care 2003;12:194-200.

30 Lynn J, Goldstein NE. Advance care planning for fatal chronic illness: avoiding commonplace errors and unwarranted suffering. Ann Intern Med 2003;138:812-8.

31 Friend T. Medical mistakes happen when safeguards fail. USA Today 1 April, 2003.

32 Adams KA GG, Searcy CA. Development of a performance model of the medical education process. Technical Report commissioned by the Association of American Medical Colleges. Washington, DC: American Institutes for Research, 2001.

33 Baker DP, Salas E, King $\mathrm{H}$, et al. The role of teamwork in the professional education of physicians: current status and assessment recommendations. Jt Comm J Qual Patient Saf 2005;31:185-202.

34 Aron DC, Headrick LA. Educating physicians prepared to improve care and safety is no accident: it requires a systematic approach. Qual Saf Health Care 2002;11:168-73.

35 Aggarwal R, Undre S, Moorthy K, et al. The simulated operating theatre: comprehensive training for surgical teams. Qual Saf Health Care 2004; 13(Suppl 1):i27-32

36 Flin R, Maran N. Identifying and training non-technical skills for teams in acute medicine. Qual Saf Health Care 2004;13(Suppl 1):i80-4.

37 Healey AN, Undre S, Vincent CA. Developing observational measures of performance in surgical teams. Qual Saf Health Care 2004;13/Suppl 1):i33-40.

38 Morey JC, Simon R, Jay GD, et al. Error reduction and performance improvement in the emergency department through formal teamwork training: evaluation results of the MedTeams project. Health Serv Res 2002;37:1553-81.

39 Thomas EJ, Sexton JB, Helmreich RL. Translating teamwork behaviours from aviation to healthcare: development of behavioural markers for neonatal resuscitation. Qual Saf Health Care 2004;13(Suppl 1):i57-64.

40 Pronovost PJ, Wu AW, Sexton JB. Acute decompensation after removing a central line: practical approaches to increasing safety in the intensive care unit. Ann Intern Med 2004;140:1025-33.

41 Gaba DM, Howard SK, Flanagan B, et al. Assessment of clinical performance during simulated crises using both technical and behavioral ratings. Anesthesiology 1998;89:8-18.

42 Halamek LP, Kaegi DM, Gaba DM, et al. Time for a new paradigm in pediatric medical education: teaching neonatal resuscitation in a simulated delivery room environment. Pediatrics 2000;106:E45.

43 Stevens DP. Finding safety in medical education. Qual Saf Health Care 2002;11:109-10.

44 Colla JB, Bracken AC, Kinney LM, et al. Measuring patient safety climate: a review of surveys. Qual Saf Health Care 2005;14:364-6.

45 Pronovost $\mathbf{P}$, Sexton B. Assessing safety culture: guidelines and recommendations. Qual Saf Health Care 2005; 14:231-3.

46 Kaplan H, Barach P. Incident reporting: science or protoscience? Ten years later. Qual Saf Health Care 2002;11:144-5.

47 Runciman WB, Sellen A, Webb RK, et al. The Australian Incident Monitoring Study. Errors, incidents and accidents in anaesthetic practice. Anaesth Intensive Care 1993;21:506-19.

48 Weingart SN, Wilson RM, Gibberd RW, et al. Epidemiology of medical error. BMJ 2000;320:774-7. 